\title{
A Fuzzy approach for the globalization of production activities
}

\author{
A. Portioli \\ Dipartimento di Economia e Produzione, Politecnico di Milano \\ Piazza L. da Vinci 32, I-20133 Milano, ITALY \\ Tel. ++3922399.2733 \\ Fax ++3922399.2700 \\ e_mail portioli@mail.ecopro.polimi.it
}

\begin{abstract}
Globalization is one of the most important and dynamic changes in the last decades. Companies can globalize in different ways, such as: exporting, licensing, starting a production activity abroad. During the last few years, there has been an increasing number of companies choosing to globalize by starting a new plant abroad. Moreover, most models presented in literature are difficult to implement in real situations, mainly due to the following reasons: (1) many models do not manage both quantitative and non quantitative information together; (2) in globalization decisions a large number of elements are involved and their relationships are complex and difficult to model mathematically; (3) some models are crispy: they divide alternatives into good and bad, giving no idea about their degree of "goodness". In this paper a Fuzzy Logic based Decision Support System that avoids these drawbacks is presented, and an implementation of the system in an European company that was undertaking a decision process for transferring abroad some production activities is described.

Results show the usefulness and effectiveness of the proposed tool.
\end{abstract}

Keywords

Fuzzy logic, globalization, production systems 


\section{INTRODUCTION}

Over the past 15-20 years globalizations increased tremendously: richest markets attract competitors from all over the world and this caused an intensification of the competitive pressure. In order to cope with this situation, many companies adopted a combination of the following two approaches (Fawcett, 1993): (1) to improve their internal manufacturing competence through the adoption of JIT and TQM principles; (2) to develop coordinated global manufacturing networks to take greater advantage of available worldwide resources.

Despite the tremendous relevance of the globalization of production activities, very little research work has been carried out on this subject, compared with JT, TQM and other approaches to improve internal competitiveness of the company (Portioli and Sianesi, 1993).

Globalization is such a complex process that it cannot be addressed as a whole, but rather has to be approached hierarchically. In Iskra et al. (1994) a literature review of globalization models is presented, and a decision process is proposed that utilizes different models at different hierarchical levels. In particular three hierarchical levels can be identified: at the first level the main issue is about what area of the company to globalize (Purchasing, Manufacturing, R\&D, Distribution), and De Toni et al. (1992) proposed a model to support such a decision. If it has been chosen to globalize part (or all) of the production activities, models such as those proposed by Fawcett (1993), McGrath and Bequillard (1989), and Ferdows (1989) help in identifying the strategic role of the overseas activities. Finally, the third level concerns the location of the production activities, and Van de Ven (1993), Vos (1991) and Haug (1992), proposed decision models to help companies in identifying the best location, minimizing such costs as production cost, transportation cost, investment cost, etc., taking into consideration also issues as the transportation system of the target country, its political stability, etc.

As for this third level, most of the models presented in literature are difficult to implement in practice because of three main drawbacks:

1. many models have difficulties in managing quantitative and non quantitative information at the same time;

2. globalization decisions involve many different elements, linked by complex relationships, that are difficult to model mathematically in a satisfactory way;

3. some models (e.g. all those based on LP) present a black and white solution, where the optimal alternative is selected (white) and all the others are black, giving no information if, for example, the second one is a very close one, or a much worse one. On the contrary managers for their decisions would appreciate better a tool that indicates the degree of goodness and badness of alternative solutions, so they can improve the solution by taking into consideration other elements also. 
Moreover, most of the models presented in literature do not explicitly help to identify what part of the production system to transfer, but they rather take as given that information.

Therefore, in this paper a Fuzzy Logic (FL) based Decision Support System (DSS) that captures qualitative aspects, allows to easily and quickly model relationships, and supplies information about the goodness of the alternative solutions, is proposed. Besides, this DSS explicitly addresses the issue of which products, and which steps of their production process to transfer abroad showing the usefulness of this tool.

The proposed DSS has been implemented in a European middle sized company and the results have been compared with the ones yielded by their traditional decisional process.

The reminder of the paper is organized as follows: in Section 2 the proposed DSS is presented, in Section 3 its implementation in a real case is described and in Section 4 the results of the proposed DSS are analyzed. In Section 5 conclusions are given.

\section{THE PROPOSED DSS}

The decisional model proposed is characterized by the following steps:

1. analysis of the products and of the processes;

2. ordering of alternatives;

3. identification of alternative worth in further investigation;

4. quantitative evaluation of alternatives and final choice.

In the following sections each step will be described.

\subsection{Analysis of products and processes}

At this step products are analyzed in order to identify the ones that can give more advantages if their production is transferred abroad. For this analysis three classes of elements are taken into consideration: market issues (e.g. evolution in the demand, position in the product life cycle, distribution and selling characteristics, etc.), cost issues (e.g. cost distribution among manpower, raw material, etc.), and physical characteristics (weight, dimensions, number of components, etc.).

Besides the analysis of the products, an analysis of the production processes is necessary. All the processes carried out by the company are broken down into activities (steps of the production process), that are then classified on the basis of a portfolio analysis along two dimensions: effectiveness and efficiency (Figure 1)

A competence activity is an activity that the company carries out efficiently thanks to its competence.

A core activity is an activity that is cardinal for the market performances of the company (thanks to this activity the company is more effective on the market). 


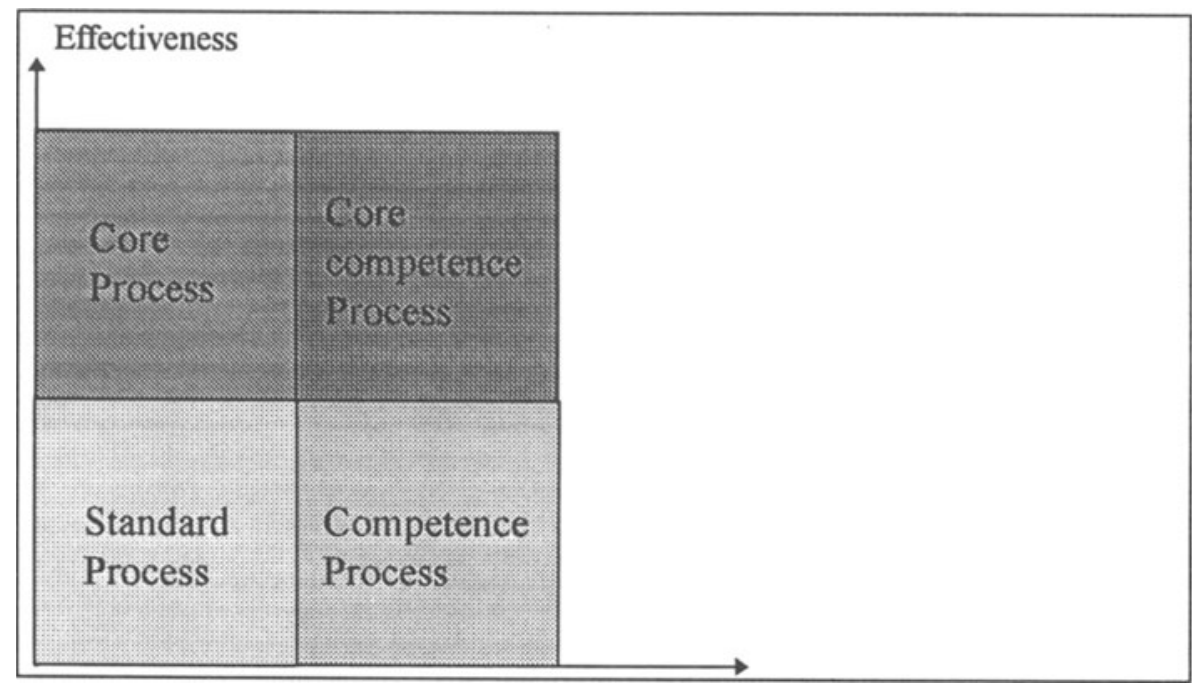

Efficiency

Figure 1: portfolio analysis

A core competence activity is an activity that is both cardinal for the market performances of the company, and is carried out efficiently.

A standard process is a process that is neither peculiar to neither the company, nor it is carried out more efficiently than by the competitors.

Non core activities can easily be transferred abroad, or even given to other companies (suppliers or subcontractors) that are more efficient in carrying them out. On the contrary, core activities are the one that the company has to concentrate on and to tightly control.

This kind of portfolio analysis is well known and it is always included in the decision models for globalization. What it is often overlooked is an analysis about links and interactions among activities. In fact, besides a portfolio analysis a cluster analysis is necessary. Sets of activities are considered to identify technological constraints (for example two activities that are to be performed in close sequence because of the high temperature of the material) and organizational constraints, in order to identify clusters of independent activities (independent clusters). In fact, it is necessary to take into consideration for transfer, not the single activity, but an independent cluster.

Transferable clusters are, therefore, independent clusters (i.e. do not have any core activity), that the products analysis has shown to be potentially profitable to transfer abroad. 
Once the set of transferable clusters, and the set of alternative countries, are identified, the next step is to order the combinations of clusters and countries, according to their "goodness".

\subsection{Ordering of alternatives}

As it has been stated above, for the managers it is much better to have all alternatives ranked on a scale, than just having the best one identified.

The problem is to choose the right scale.

Two main factors affect the goodness of the choice of what to transfer abroad and where: (1) adequacy to the transfer, and (2) fulfillment of the company's objectives.

Adequacy to the transfer is very difficult to estimate, because it is due to many different aspects, most of which are not quantifiable. In particular we have taken into consideration:

- technical complexity;

- affinity with non transferable functions;

- additional costs;

- distance from the market.

Each of these categories is further detailed in subcategories. For example the technical complexity is divided into product complexity, process complexity and environmental conditions. Each transferable cluster is given an evaluation on each one of the subcategories, for each alternative country (or location, if more than one alternative is considered for each country). In fact, such evaluation is strongly location dependent. For example, a production process can be evaluated with a high process complexity, if the location under consideration is China, but with a medium process complexity if the location under consideration is the Czech Republic, because of the different technology development and average manpower training of the two countries.

All these subcategories are difficult to quantify and are linked one another by a complex relationship that is impossible to represent mathematically. A lexical description is much easier, much faster, and even closer to reality. Therefore, a FL model has been developed to capture this capital aspect (managers can easily describe these relationships by means of IF.... THEN... statements).

On the contrary, as for the fulfillment of the company's objectives, a multi objective function has been preferred, because it allows a straightforward understanding and more transparency.

The objectives of a company when transferring part of the production abroad can be one or more of the following (Ferdows, 1989):

- access to low cost production input factors;

- proximity to the market;

- use of local technological resources; 
- control and amortization of technological assets;

- pre-emption of competition.

Each one of these objective has been further detailed (e.g. low cost production input factors are: raw material, energy, money, manpower) to help in better estimate the value, for each transferable cluster and each alternative country. Each company sets the weights for the objectives so to get a customized overall objective function.

\section{3 identification of alternative worthing further investigation;}

By means of step 1 and step 2 we obtain the position of the different alternative on the scale of the adequacy to the transfer, and on the scale of the fulfillment of the company's objectives, for each country. It is therefore possible to chart the transferable clusters on a two dimensional diagram (Figure 2)

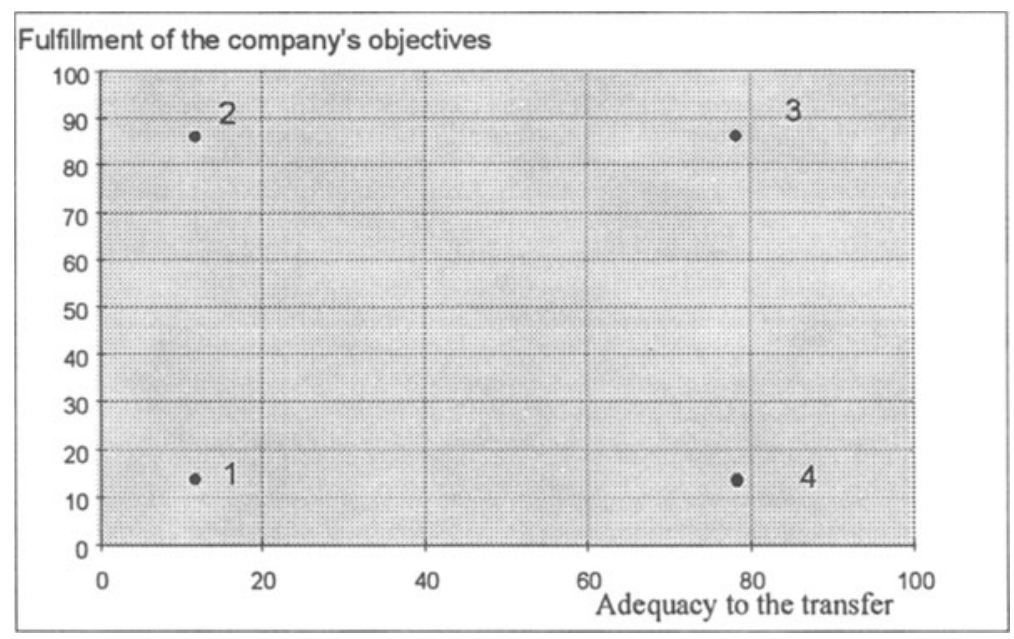

Figure 2: Example of the valuation chart for alternative transferable clusters for a certain country.

Alternatives in position 3 present a high value for both fulfillment of the company's objectives and for the adequacy to transfer, and so deserve further analysis. On the contrary, alternatives in position 1 can be disregarded because are difficult to transfer and give a low contribution to the fulfillment of company's objectives. Also, alternatives in position 4 have low interest. Finally, alternatives in position 2 could cause difficulties if transferred, but could yield a large advantage to the company if the transfer is correctly implemented: they can be seen as opportunities that require particular attention and need a deeper analysis. 


\section{4 quantitative evaluation of alternatives and final choice.}

For the alternative that the previous step has shown worth further consideration, an investment analysis is carried out in order to have monetary data also. The amount of the investment necessary is evaluated, the cash flows are estimated and a financial plan is formulated to decide which alternative to actually implement, and when. This is a standard analysis, and therefore it will not be further described.

The proposed DSS has been first tested for internal consistency by applying the model to different benchmark situations (cases with well defined characteristics, for which the good solutions were known and clear). Then, it has been implemented in a real case, that is described in the next section.

\section{THE GLASS-WORKER COMPANY CASE}

The Glass-worker company (for confidential reasons we use a fictional name) is a middlesized European company, that employs about 800 workers, with a turnover of about 140 million dollars a year. The industries it works for are: Glass, Optoelectronics, Quartz and Automotive. The Glass-worker produces some 15\% of the world market of capsules for electronics devices (e.g. electronic airbag sensors), and some products show a relevant increase in the turnover.

The market was facing an increasing degree of competition, mainly due to the entry of new Asian competitors offering similar products at lower costs. The management had decided to respond by transferring abroad part of the production, so they started to analyze the problem and evaluate different alternatives.

After a first analysis, the alternative countries taken into consideration are: China, Czech Republic, and Malaya.

After 8 months the decision process was almost at the end, so we thought it a good opportunity to undertake a parallel process with other managers of the company, adopting the proposed tool, in order to prove its practical feasibility and its advantages over the traditional one.

The products analysis and the process analysis have allowed as to identify the products that could get considerable advantages if transferred abroad, and the clusters that do not contain any core activity. A further constraint set by the management is that a transferable cluster must contain all activities necessary to complete an end product, or a component that is sellable (i.e. that is not only for internal production, but that has its own market). Therefore, in this case a transferable cluster is also identified by the item it realizes (Table 1 presents the items corresponding to the transferable clusters).

Then a manager of the Glass-worker was asked to use the FL based software for determining the adequacy to transfer of the transferable clusters, for each one of 
the 3 alternative countries, indicating a value of very low, low, medium, high, very high, for each of the subcategories building up the adequacy.

As for the fulfillment of the company's objectives, management gave the weights shown in Table 2.

Table 1: Transferable clusters/items

\begin{tabular}{clc}
\hline Number & \multicolumn{1}{c}{ Description } & Code \\
\hline 1 & Glassdust & AZ851 \\
2 & PR for Reed & WQ882 \\
3 & Transistor TO 5/ TO39 & ZA854 \\
4 & DIP-Socket & KW850 \\
5 & SQ-Floor & WF870 \\
6 & Top of Actuator for CFZ & XS863 \\
7 & Top of Actuator for Sensors & KB866 \\
\hline
\end{tabular}

Table 2: Objectives' weight for the Glass-worker case.

\begin{tabular}{ll}
\hline Objective & Weight \\
\hline access to low cost production input factors & 0.8 \\
proximity to the market & 0.2 \\
use of local technological resources & 0 \\
control and amortization of technological assets & 0 \\
pre-emption of competition & 0 \\
\hline
\end{tabular}

All transferable clusters have been taken into consideration according with this overall objective function and an evaluation chart has been derived for each country. In Figure 4 the Czech Republic's evaluation chart is reported. 


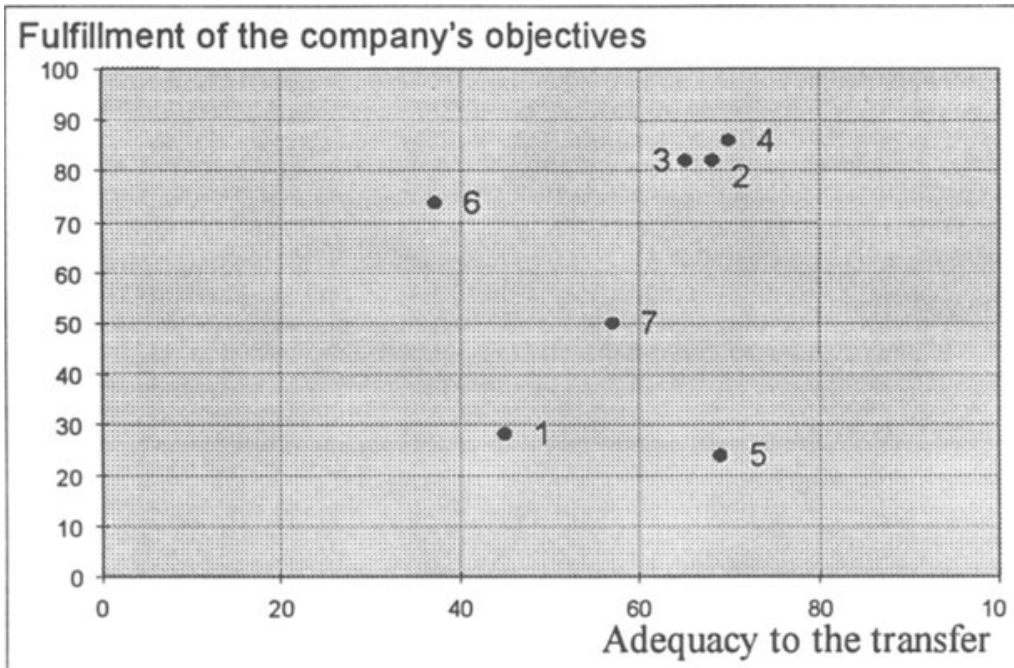

Figure 3: the valuation chart for alternative transferable clusters for the Czech Republic

From the chart it can be seen that items 2, 3 and 4 should be transferred, item 5 has a lower priority (it fulfills less the company's objectives), but it is interesting also, and should give little problem if transferred. Item 6 would give a great advantage if transferred, but has also a low adequacy to transfer, this is due to its high affinity with non transferable functions (in particular frequent and intense information exchange with the R\&D).

Comparing the evaluation charts for the different countries it can be seen that the Czech Republic is the one that yields the best values. The main reasons can be found in that, on the one hand China has higher transportation costs, low quality standard for raw materials and does not have an internal market for the items considered. On the other hand, Malaya has a heavier fiscal policy, and a harsh environmental regulation, that would require costly additional investments.

At this point results have been compared with the one achieved by means of the traditional decision process.

\section{ANALYSIS OF RESULTS}

The Glass-worker company's traditional decision process had selected for transferring items 3, 4 and 6 . A deeper analysis has been carried out together with 
the Glass-worker company's management, in order to understand the reasons for the differences:

1. the proposed DSS suggests item 2 as a good one to transfer, but the Glassworker's management notices that it is an item that in the near future will be replaced by a new one, adopting a different technology. This aspect would have been captured in the quantitative analysis, when estimating the cash flows;

2. items 7 and $\mathbf{5}$ had not been selected for transfer by the Glass-worker's management because improvement in performances was mainly pursued by cutting production costs $(0.8)$, while proximity to the market and the opportunity to increase sales was not considered very important $(0.2)$. Therefore, in meeting after meeting all choices discussed, focused more and more on costs ("it is not possible to keep in mind always everything: you have to focus on main points"). Nevertheless, particularly item 7, showed a great selling potential, thus increasing significantly the fulfillment of the company's objectives (the weight of the objective is low, but the selling potential of 7 is very high). The proposed DSS, unlike the traditional process, had no problems in considering also low priority objectives, thus allowing the capture of more opportunities. The Glass-worker company's management accepted the suggestion of the FL tool and added item 7 to the pool of items to be further considered for transferring.

3. item 6 has been selected for transferring by the Glass-worker's management, but the proposed DSS highlights a low adequacy to transfer (because of its affinity with non transferable functions). The management agreed with the results of the DSS. They decided to confirm transfer of item 6 , but to launch a study to activate all possible actions so to guarantee the intense information transfer that the proposed DSS highlighted to be necessary (e.g. electronic connections with the new site, video conferencing, pc networks, etc.).

When compared with the traditional Glass-worker company's decision process, the proposed DSS shows to be more structured and allows making explicit relationships among characteristics that otherwise could remain unexpressed and could: on the one hand increase the degree of subjectiveness (not all terms are clear to everybody), on the other hand delay the decision process (a good part of the meetings is lost in focusing the discussion and in clarifying, or reminding, the interrelations among the considered elements). Therefore, the Glass-worker company's management appreciated the ability of the tool to shorten the decision process (the traditional one took 10 months, using the FL tool 2 months were enough), and to reduce the degree of subjectiveness. This last point has been further proven by undertaking the decision process with different persons (sharing similar knowledge about the company's products, processes and objectives) and showing that the results were quite similar. 


\section{CONCLUSIONS}

In this paper a new system for supporting the decision process of companies that want to globalize their production system has been presented, and its application to a real case has been described.

Managers of the company where it has been implemented appreciated very much this method, because it is able to:

- manage both quantitative and non quantitative data;

- manage uncertain and subjective information;

- cope with a high number of interrelations among parameters, that are not easy to model mathematically (if not impossible), but, on the contrary, can be easily treated in the proposed DSS by means of IF, THEN statements;

- present the degree of "goodness" of each alternative, and not just to point out the best one.

Moreover, the managers involved in the test appreciated particularly the ability of the tool to consider at the same time more than one objective, thus not forgeting low priority objectives also. Finally, reduction in the time to perform the decision process and in its degree of subjectiveness were also considered major advantages of the proposed tool.

\section{REFERENCES}

De Toni A., Filippini R., Forza C. (1992) Manufacturing strategy in global markets: an operations management model. International Journal of Operations and Production Management, 12, 4, 7-18.

Fawcett, S.E. (1993) Conversion system cost characteristics in global manufacturing: the maquiladora example. International Journal of Production Research, 31, 3, 647-664.

Ferdows K. (1989) Mapping international factory networks, in Managing international manufacturing (ed. K. Ferdows), North-Holland, Amsterdam.

Haug P. (1992) An international location and production transfer model for high technology multinational enterprises. International Journal of Production Research, 30, 3, 559-572.

Iskra R., Marinoni A., Portioli A., Sianesi A. (1993) Analysis of internationalization models. Advanced topics on production systems design and management. Varenna (Lecco), ITALY, June 1-4 (in Italian).

Mc Grath M.E., Bequillard R.B. (1989) International manufacturing strategies and infrastructural considerations in the electronics industry, in Managing international manufacturing (ed. K. Ferdows), North-Holland, Amsterdam. 
Portioli A. and Sianesi A. (1993) Globalization of the companies and of their production systems.To be an Industrial Engineer today... and tomorrow? ANIMP XX national conference, Capri (Naples) 14-16 October (in Italian).

Van de Ven A.D.M. and Ribbers A.M.A. (1993) International logistics: a diagnostic method for the allocation of production and distribution facilities. International Journal of Logistics Management, 4, 1, 67-83.

Vos G.C.J.M. (19921 A production-allocation for international manufacturing strategy. International Journal of Operation and Production Management, $11,3,125-134$.

\section{BIOGRAPHY}

Alberto Portioli carries out his research activity at the Dipartimento di Economia e Produzione of the Politecnico di Milano in Italy. His research interests are in the areas of production systems design, operations management, manufacturing strategy and quality. On these subjects he published papers on international journals as International Journal of Production Research, Production Planning and Control, and others. Alberto Portioli also teaches Operations Management in an undergraduate course and in a MBA course of the Politecnico di Milano. 